\title{
In situ transmission electron microscopy studies of shear bands in a bulk metallic glass based composite
}

\author{
E. Pekarskaya, C.P. Kim, and W.L. Johnson \\ Keck Laboratory of Engineering Materials, California Institute of Technology, \\ Pasadena, California 91125
}

(Received 16 March 2001; accepted 8 June 2001)

\begin{abstract}
In situ straining transmission electron microscopy (TEM) experiments were performed to study the propagation of the shear bands in the $\mathrm{Zr}_{56.3} \mathrm{Ti}_{13.8} \mathrm{Cu}_{6.9} \mathrm{Ni}_{5.6} \mathrm{Nb}_{5.0} \mathrm{Be}_{12.5}$ bulk metallic glass based composite. Contrast in TEM images produced by shear bands in metallic glass and quantitative parameters of the shear bands were analyzed. It was determined that, at a large amount of shear in the glass, the localization of deformation occurs in the crystalline phase, where formation of dislocations within the narrow bands are observed.
\end{abstract}

\section{INTRODUCTION}

A new class of materials emerged with the discovery of the multicomponent systems with exceptional glassforming ability, which made possible the synthesis of metallic glasses at large thicknesses. ${ }^{1,2}$ Bulk metallic glasses have unusual mechanical properties, such as high elastic limit, up to $2 \%$, and very high yield stress of $0.02 Y$ ( $Y$ is Young's modulus) but exhibit almost no global plasticity. ${ }^{3}$ Two regimes of plastic deformation have been observed, viscous or homogeneous deformation, which occurs at high temperatures and low strain rates, and inhomogeneous flow at low temperatures and high strain rates. ${ }^{4}$ Inhomogeneous deformation manifests itself in the formation of shear bands at a $45^{\circ}$ angle with respect to the applied stress in uniaxial loading. Deformation mechanisms of metallic glasses attracted a lot of attention from both theoretical and experimental sides. ${ }^{4-10}$ Most of the experimental work, however, was done using scanning electron microscopy (SEM). Transmission electron microscopy (TEM) studies of shear bands were not very successful so far due to the relatively small structural changes in the shear bands, frequently undetectable by TEM. The most successful approach in studying shear bands in metallic glasses turned out to be in situ straining experiments or deformation of thin films of material without successive thinning. ${ }^{11,12}$ Propagation of a shear band in a thin film creates a step at the surface and changes the mass-thickness contrast in the TEM in the region of the shear band.

This paper reports the TEM analysis of shear bands in a two-phase material, $\mathrm{Zr}_{56.3} \mathrm{Ti}_{13.8} \mathrm{Cu}_{6.9} \mathrm{Ni}_{5.6} \mathrm{Nb}_{5.0} \mathrm{Be}_{12.5}$, which consists of amorphous and crystalline phases. Such multiphase materials and composites based on a glassy matrix have been developed in attempt to improve ductility and toughness of metallic glasses. ${ }^{13,14}$ The presence of the second phase particles of crystalline phase was found to hinder shear band propagation and promote formation of multiple shear bands. ${ }^{13}$ The goal of our study was to understand the deformation mechanisms of such multiphase material using TEM.

\section{EXPERIMENTAL}

The alloy was prepared by the arc melting of a mixture of constituent elements, with purity of 99.7 at.\% or better, in a Ti-gettered argon atmosphere on a water-cooled $\mathrm{Cu}$ crucible. The specimens were flipped over and remelted several times to promote homogeneity. The specimens for TEM were prepared by ion milling. In situ straining (tensile) experiments were performed at the Center for Microanalysis of Materials in the University of Illinois at Urbana-Champaign, IL. A JEOL 4000EX TEM operating at $300 \mathrm{kV}$ was used. The experiments were performed at room temperature. The test was interrupted several times to analyze the changes in the microstructure during different stages of deformation. In addition, a Philips EM430 TEM operating at $300 \mathrm{kV}$ (at California Institute of Technology) was used to study the specimens after deformation.

\section{RESULTS AND DISCUSSION}

\section{A. Shear bands in metallic glass}

The microstructure of the alloy prior to deformation is presented in Fig. 1. The alloy consists of the amorphous matrix and crystalline phase, $\beta$, which forms a dendritic structure. Most of the dendrites have a bcc structure with lattice parameters of $3.5 \AA$. Extra reflections in diffraction patterns indicate ordering [see inset in Fig. 1(a)], as well as a "spotty" contrast, which most likely originates 


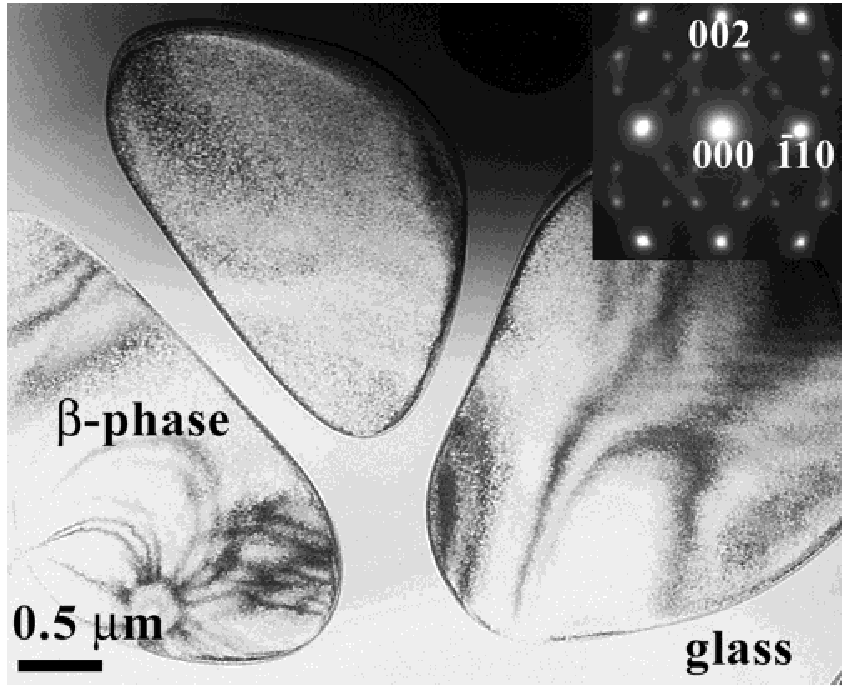

(a)

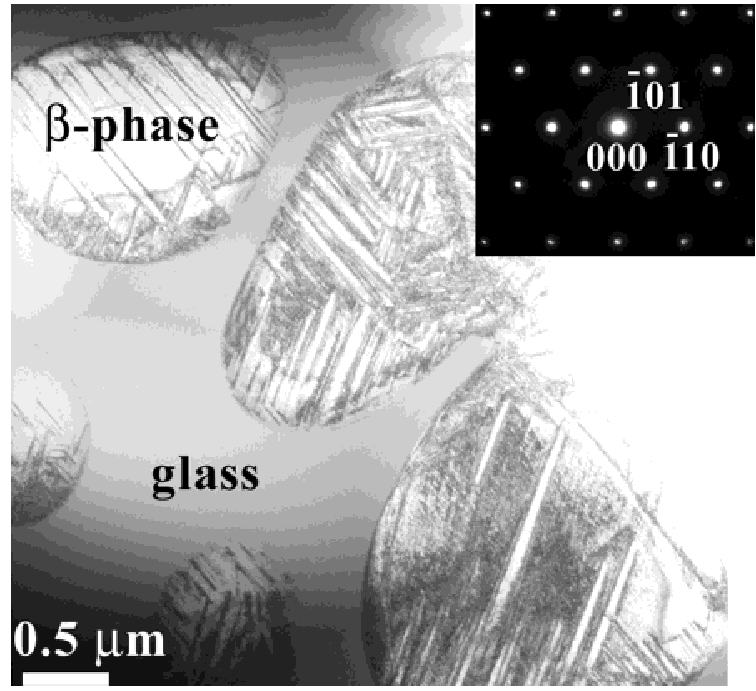

(b)

FIG. 1. (a) TEM micrograph of the microstructure of the composite material. The diffraction pattern in the insets is a [110] zone axis of the $\beta$-phase. Extra reflections indicate ordering. (b) TEM image of the $\{112\}$ twins in the $\beta$-phase. The diffraction pattern is a [111] zone axis. Twin and matrix reflections coincide.

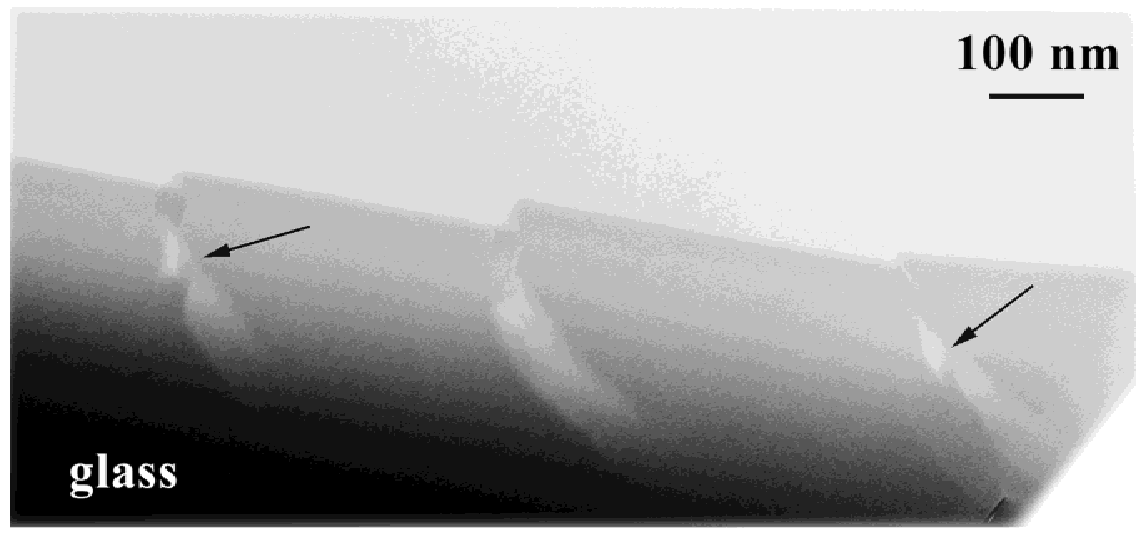

(a)

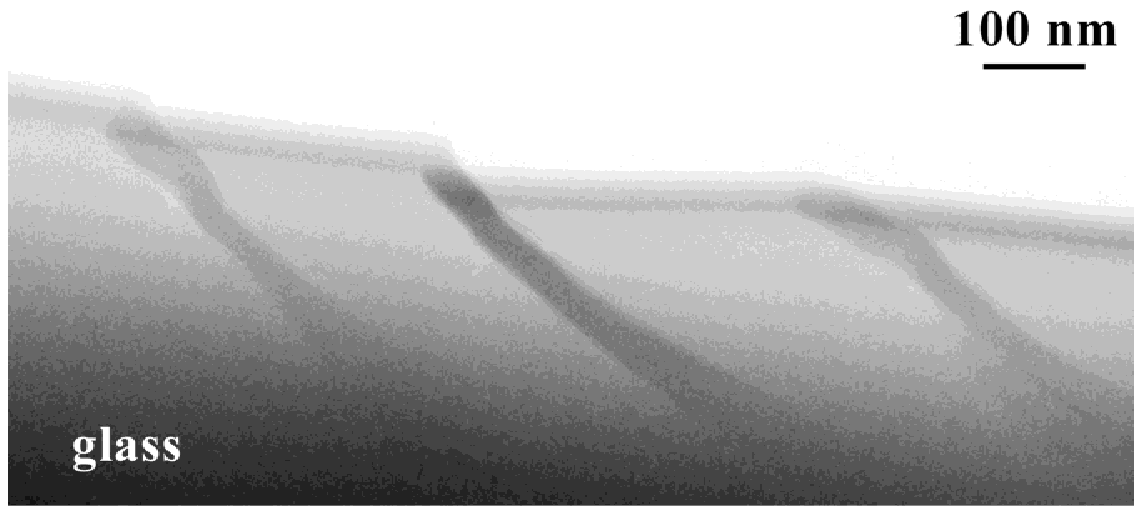

(b)

FIG. 2. Bright-field images of the shear bands formed during in situ deformation at the edge of the specimen. (a) and (b) represent the same area of the specimen at different tilt angles. Shear band branching is indicated by arrows. 
from the ordered domains. Some $\beta$-phase dendrites contain twins on $\{112\}$ planes [Fig. 1(b)]. The presence of twins indicates instability of the bcc structure. This is related to the fact that some dendrites have an orthorombic face-centered structure $(a=4.8 \AA, b=4.7 \AA$, $c=$ $4.4 \AA$ ) with twins on $\{111\}$ planes, as determined by electron diffraction analysis This phase could be a product of the martensitic transformation of the $\beta$-phase.

During the in situ tensile tests, the failure of the specimens occurred in the direction normal to the applied stress. A localized deformation was observed mainly in the vicinity and ahead of the cracks, where shear bands were formed.
Figure 2 shows bright-field TEM images of the shear bands which were formed at the edge of the specimen. Depending on the orientation of the shear direction with respect to the electron beam, the shear band appears either lighter [Fig. 2(a)] or darker [Fig. 2(b)] compared to the surrounding material. ${ }^{12}$ Shear band branching frequently occurs, which can be seen in Fig. 2 .

In most cases in this study, dark-field imaging of the shear bands, with the objective aperture centered on the first halo, produced very faint, almost undetectable contrast. Therefore, in this work, analysis of shear bands was done mainly using bright-field imaging conditions. This contradicts the observations made by Donovan

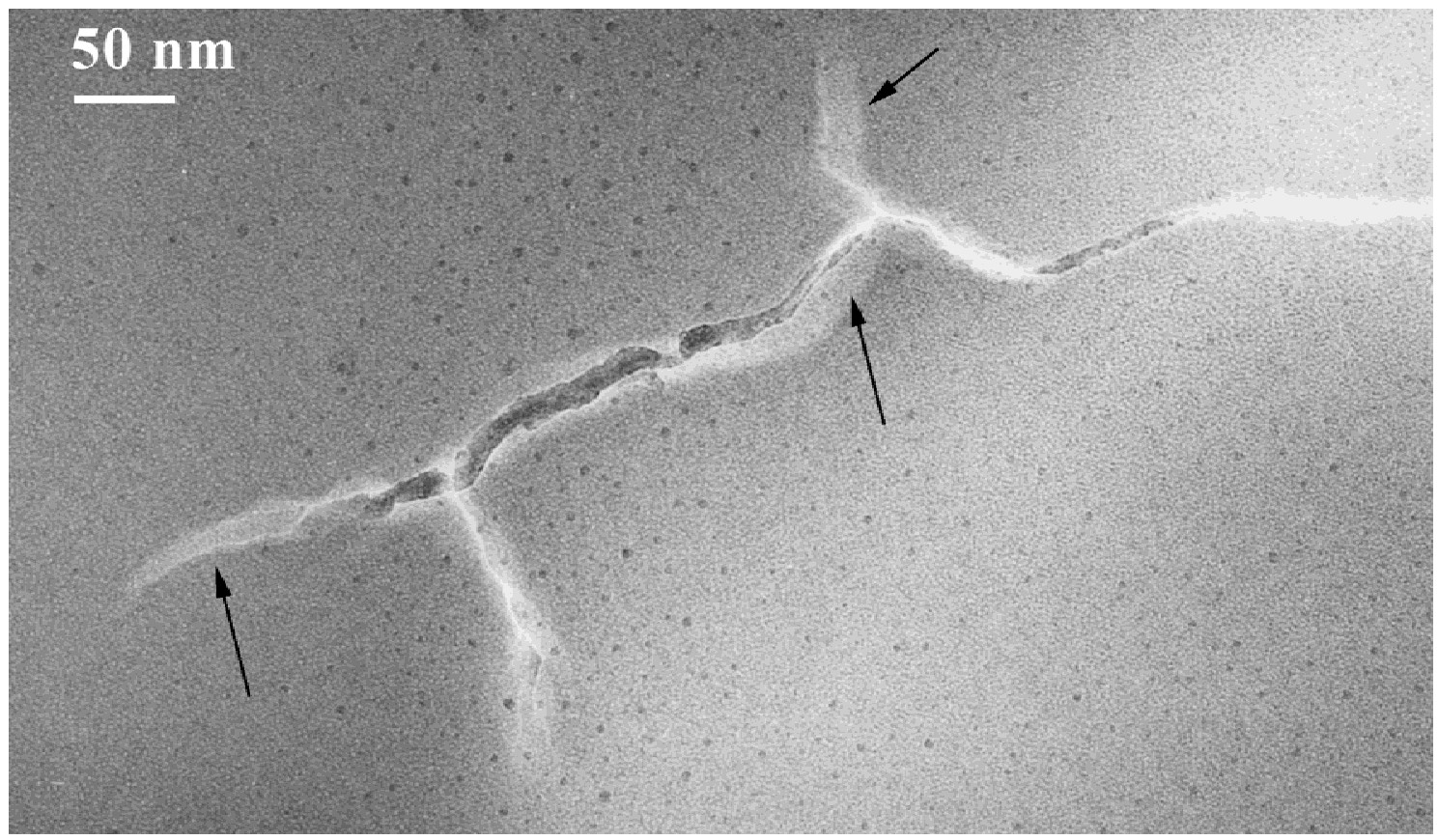

(a)

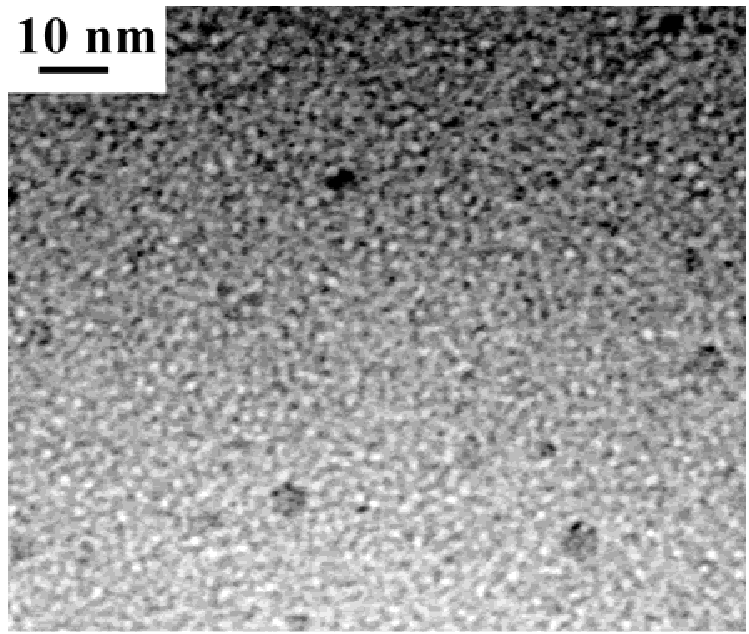

(b)

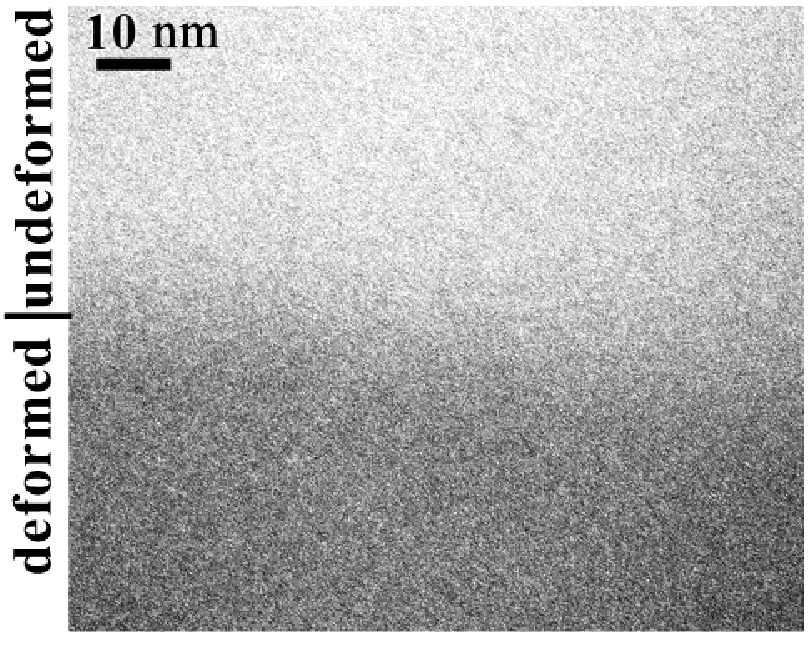

(c)

FIG. 3. (a) Crack propagating in the glassy phase. Shear bands can be observed at the tip of the crack, indicated by arrows. Magnified bright-field (b) and dark-field (c) images show the structure in the deformed and undeformed regions of the material. 
et $a l .{ }^{11}$ and Glezer et al., ${ }^{12}$ who found that the contrast from shear bands in the dark-field images was more pronounced.

The changes in the microstructure can sometimes be detected in the shear bands in the glassy phase. Figure 3(a) shows a crack propagating in the glassy matrix. At the tip of the crack and crack branches, as well as in the narrow region around the crack, a sheared region can be observed, represented by a lighter contrast compared to the rest of the material. A magnified bright field image in Fig. 3(b) shows a less dense structure in the shear band compared to the undeformed material. In the dark-field images with the objective aperture centered on the first halo [Fig. 3(c)], the shear band appears dark which means that this region is thinner compared to the surrounding material (less scattered electrons). No noticeable changes could be detected in the electron diffraction patterns, probably due to the fact that the diffraction patterns with the available selected area apertures give combined information from the deformed and undeformed regions. Nonetheless, the bright-field and dark-field images suggest the reduction in density/ thickness of the material in the shear band.

Shear bands frequently change their "plane", which results in the change of the contrast from light to dark. The thickness of the shear band can be measured at the point at which the shear band is oriented edge-on. ${ }^{12}$ (The thickness of the shear band is defined as the dimension in the direction orthogonal to the slip "plane", where as the width is the size of the shear band in the shear "plane".) It should be noted that only the width of shear bands is possible to measure from the SEM images, whereas TEM enables measurements of the other dimension. This is illustrated in Fig. 4. Two shear bands can be seen in this image formed in the glassy phase, acting as stress concentrators at the glass/crystal interface. The point, where the shear band is "edge-on", is indicated by the arrowhead. The maximum thickness of the shear bands, measured this way in TEM, do not exceed $10 \mathrm{~nm}$. Donovan and Stobbs also reported 10- and 20-nm thickness of shear bands formed in $\mathrm{Fe}_{40} \mathrm{Ni}_{40} \mathrm{~B}_{20}$ metallic glass. ${ }^{11}$ The projected width of shear bands that are formed during our in situ experiments is around $120-200 \mathrm{~nm}$. These are slightly smaller values than that obtained from the SEM images (around 200-300 nm). However, it should be borne in mind that the parameters of shear bands can be effected by the thickness of the deformed material. Also, in cases when shear bands are not parallel to the specimen surface, we can measure only the projected width of the shear band and this is determined by the specimen thickness.

\section{B. Slip transfer between the glassy and the crystalline phases}

An interaction of the shear bands with the $\beta$-phase dendrites is illustrated in Fig. 5. In the top left corner of the micrograph a crack is visible, which was formed along the path of the shear band, as shown by video recording of the straining experiments. A very interesting phenomenon can be observed here, namely localization of the deformation in the crystalline phase, evidently imposed by the geometry of slip in the amorphous matrix. This result is in an excellent agreement with the SEM analysis of shear bands in this alloy, which suggested the localization of deformation in the crystalline dendrites. ${ }^{13}$

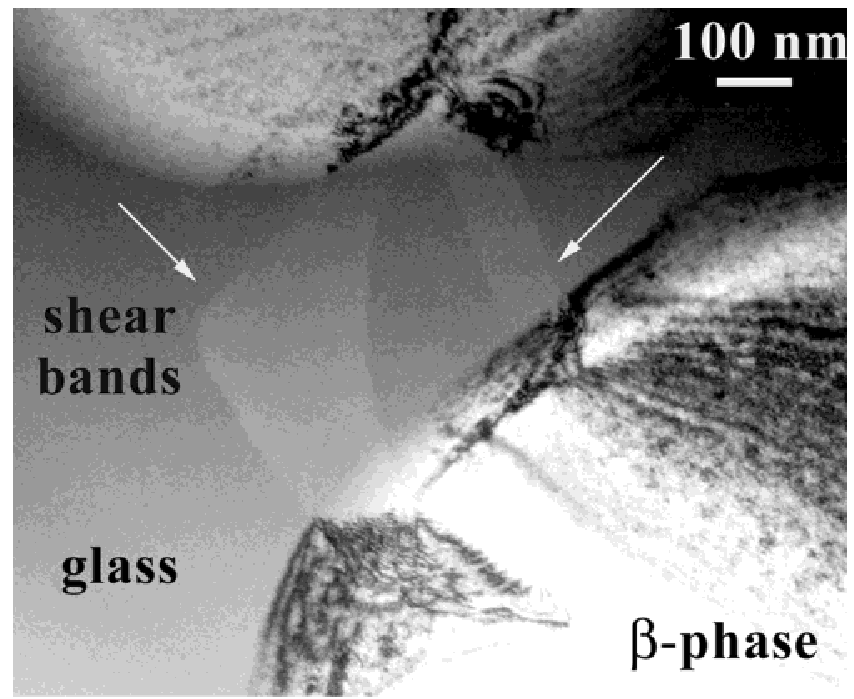

(a)

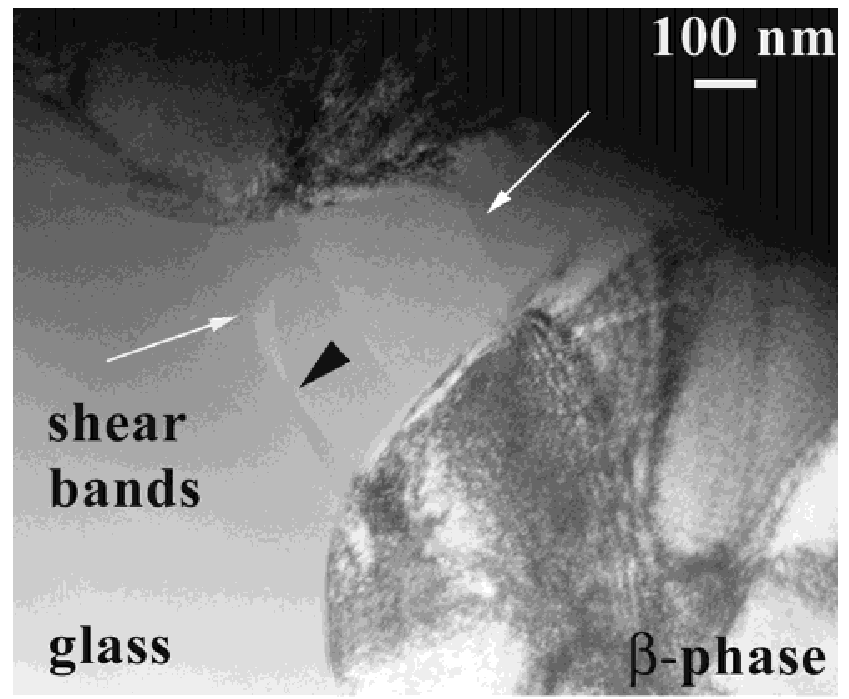

(b)

FIG. 4. TEM images of the same area at different tilt angles, illustrating changes in the shear band contrast. Shear bands are indicated by white arrows. The point where the shear band is in the "edge-on" position is marked by the black arrowhead (b). 
Tilting experiments revealed that dislocations are responsible for the localized deformation in the crystalline phase. This is illustrated in Fig. 6, where dislocations formed in the shear band in the $\beta$-phase can be observed. Analysis of Burgers vectors suggests that they are of $\langle 111\rangle$ type.

However, localization of deformation in the $\beta$-phase occurs only at a large amount of shear in the amorphous phase. Small amount of shear leads to the delocalization of deformation in the crystalline phase. The later is shown in Fig. 7, where shear bands are formed in the region of the crack tip. Slip transfer from the amorphous matrix to the crystalline phase results in the formation of dislocations in the $\beta$-phase, which are not confined to a band.

\section{Amount of shear}

When the individual dislocations can be easily resolved in the deformed region of the $\beta$-phase (e.g., Fig. 7), it is possible to estimate the amount of shear produced. The magnitude of shear is equal to $n b$, where $n$ is the number of dislocations formed due to the slip transfer from the glassy phase to the $\beta$-phase and $b$ is a Burgers vector of the dislocations. In cases such as in Fig. 7, it gives us the value of about $4 \mathrm{~nm}$. Such cases are associated with the delocalization of deformation in the crystalline phase.

The magnitude of shear can be also measured by the shear displacement at the interface, when the direction of shear does not coincide with the electron beam. An example of such offset produced by the shear bands can be seen in Figs. 2 and 6, where much stronger localization of deformation is observed. These measurements give the value of $20-50 \mathrm{~nm}$, which is larger than that obtained above from the dislocation analysis in the $\beta$-phase. Analysis of the contrast produced by shear bands in TEM images (strong or faint) and the degree of localization of deformation in the $\beta$-phase in these two cases support this result. The larger the amount of shear in the glass the more pronounced is the localization of deformation in the crystalline phase.

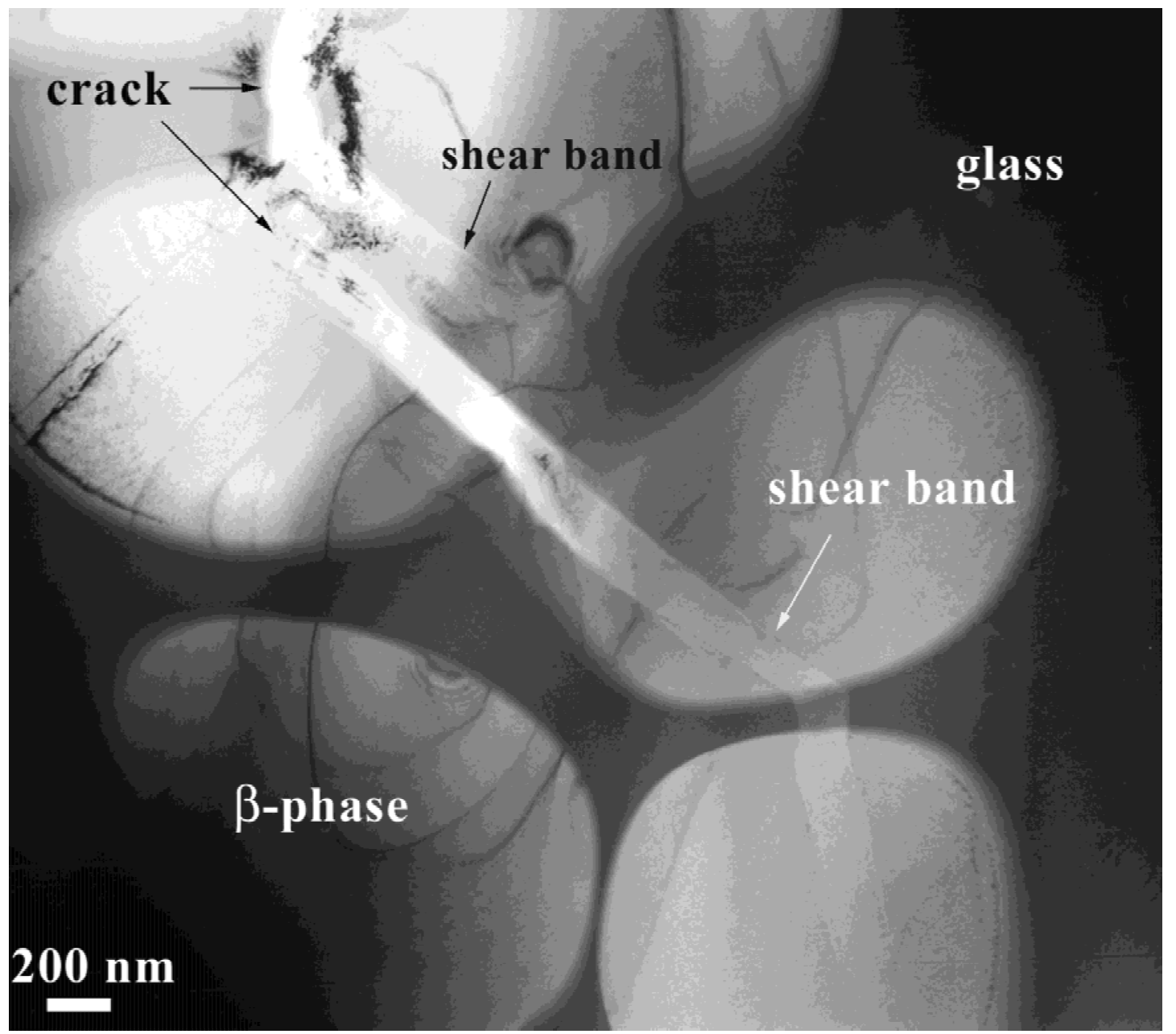

FIG. 5. TEM image of the shear band propagating through the two-phase region. Localization of deformation is observed in the $\beta$-phase. 


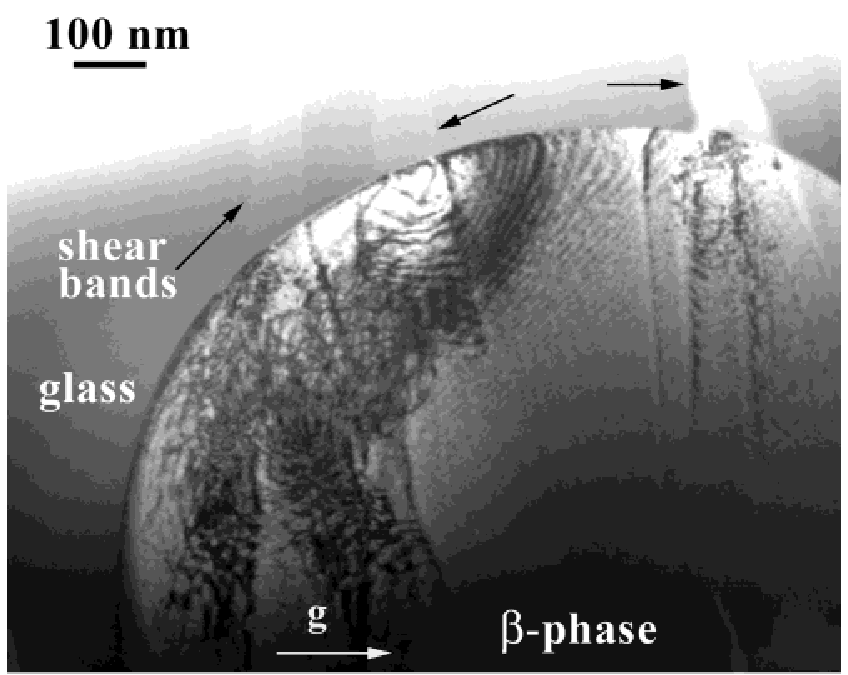

(a)

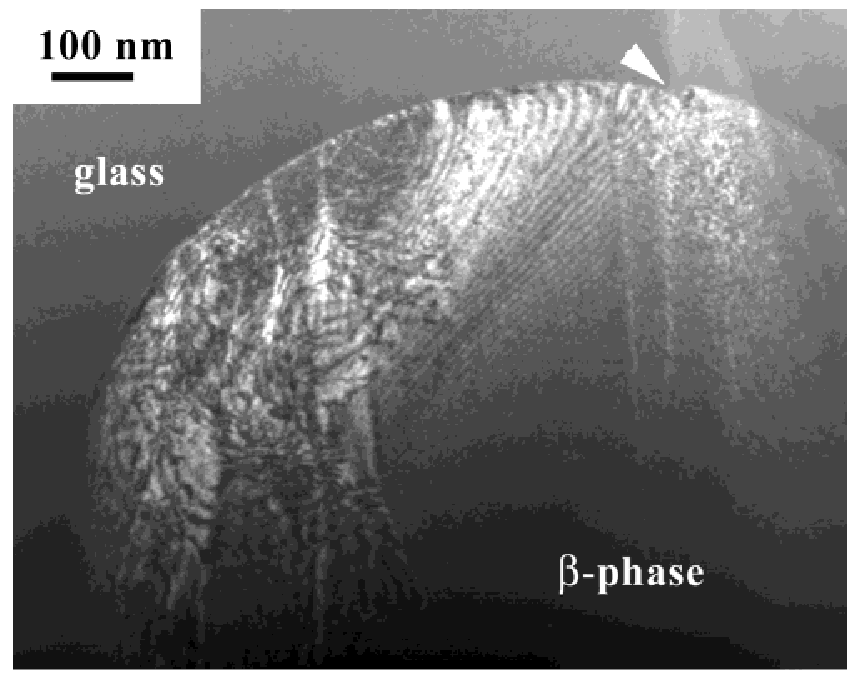

(b)

FIG. 6. (a) Bright-field and (b) dark-field ( $g=110$ ) images, illustrating the slip transfer across the interface in the bulk metallic glass based composite. Shear bands are indicated by arrows in (a). A shear offset, marked by the arrowhead in (b), is visible at the glass/crystal interface.

Measurements of the shear offsets using SEM images give larger values, from $500 \mathrm{~nm}^{13}$ up to $2 \mu \mathrm{m} .{ }^{6}$ Also, the shear displacement, which we measured in TEM in the specimens deformed in a bulk form, is around $150 \mathrm{~nm}$. This result again indicates the influence of the film thickness on the dimensions of the shear bands.

\section{SUMMARY}

TEM studies of shear bands formed during in situ straining in a two phase composite, based on a bulk metallic glass, are presented in this paper. Contrast produced by the shear bands was found to be more pronounced in the bright-field images rather than in the dark field. It has been observed that a shear band path is

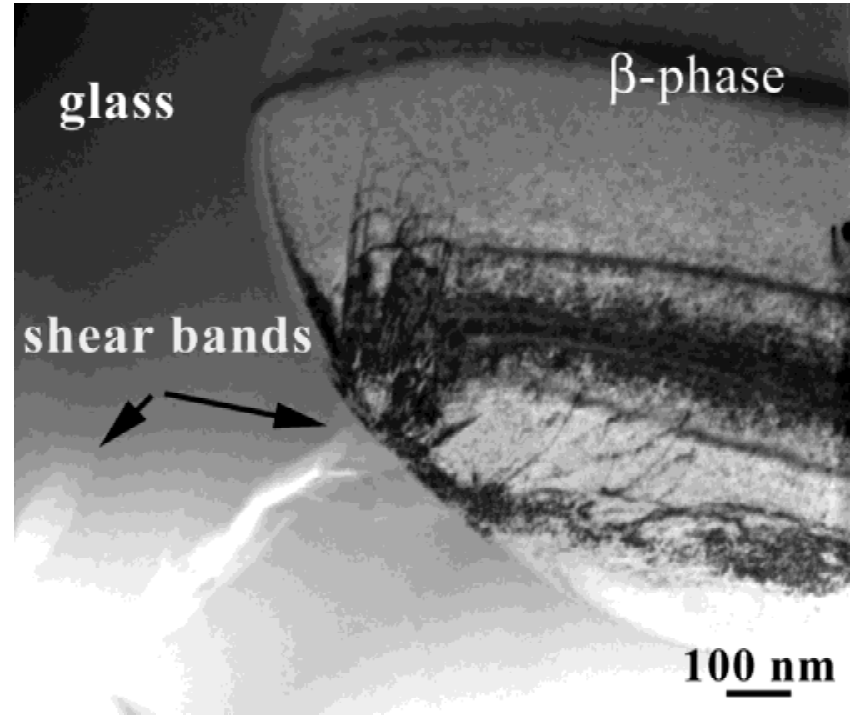

FIG. 7. TEM micrograph illustrating delocalization of deformation in the $\beta$-phase at a small amount of shear in the glass.

not confined to one "plane". The shear bands are 120-200-nm wide and around 10-nm thick. Localization of deformation was observed in the crystalline $\beta$-phase, which is in an excellent agreement with the SEM analysis of shear bands in the same material. It is established that dislocations are responsible for the deformation of the $\beta$-phase.

\section{ACKNOWLEDGMENTS}

This work was supported by the Materials Research Science and Engineering Center (MRSEC) Program of the National Science Foundation, under award No. DMR-0080065. E.P. gratefully acknowledges Prof. I.M. Robertson for assistance with in situ straining experiments.

\section{REFERENCES}

1. A. Inoue, T. Zhang, and T. Masumoto, Mater. Trans. JIM 31, 177 (1990).

2. A. Peker and W.L. Johnson, Appl. Phys. Lett. 63, 2342 (1993).

3. W.L. Johnson, MRS Bull. 24, 42 (1999).

4. A.S. Argon, J. Phys. Chem. Solids 43, 945 (1962).

5. F. Spaepen and D. Turnbull, Scr. Met. 8, 563 (1974).

6. P.G. Zielinski and D.G. Ast, Philos. Mag. A 48, 811 (1983).

7. E.D. Tabachnikova, P. Diko, V. Ocelik, and P. Duhaj, Solid State Phenom. 35-36, 569 (1994).

8. T.C. Hufnagel, P. El-Deiry, and R.P. Vinci, Scr. Mater. 43, 1071 (2000).

9. P. Lowhaphandu, L.A. Ludrosky, S.L. Montgomery, and J.J. Lewandowski, Intermetallics 8, 487 (2000).

10. A. Inoue, Intermetallics 8, 455 (2000).

11. P.E. Donovan and W.M. Stobbs, Acta Met. 29, 1419 (1981).

12. A.M. Glezer and B.V. Molotilov, Structure and mechanical properties of amorphous alloys (Metallurgiya, Moskva, Russia, 1992), p. 90 (in Russian).

13. C.C. Hays, C.P. Kim, and W.L. Johnson, Phys. Rev. Lett, 84, 2901 (2000).

14. R.D. Conner, R.B. Dandliker, and W.L. Johnson, Acta Mater. 46, 6089 (1998). 